\title{
Gathering Baseline Data for a Rural Cataract Surgical Outreach Service in Igabi District, Nigeria
}

\author{
M Mansur Rabiu MSc, CEH, FWACS, FMCOph; CP Ozemela, DI Apiafi \\ National Eye Centre, Kaduna, Nigeria
}

\begin{abstract}
Background: In an effort to plan for effective community-based cataract surgical services in Igabi district of northern Nigeria, the National Eye Centre, Kaduna (a tertiary eye hospital) conducted a population-based cross sectional survey in the district. The survey was conducted in order to generate baseline data on cataract blindness in the district.
\end{abstract}

Methods: A total of 2044 people out of 2400 eligible people were examined. The study population was chosen by two-stage cluster random sampling. In the first sampling stage, 30 clusters/wards were randomly chosen, while in the final stage, 80 people who were 40 years and over were selected in each ward. Each of the selected persons had visual acuity recorded for both eyes. Those with vision less than 3/60 in the better eye were assessed for cataract. People with cataract were asked why they had not sought medical attention.

Results: A blindness prevalence of $4.1 \%$ (95\% CI 3.2\%-5.0\%) was found among the sampled population. Cataract was responsible for $52.3 \%$ of the blindness. Thus, the prevalence of cataract blindness was found to be $2.2 \%$ (95\% CI 1.6\%-2.8\%). The cataract surgical coverage (people) was $12.8 \%$ and the couching coverage (people) was $10.3 \%$. The main barrier to seeking cataract surgery was the inability to afford the cost of service (61.5\%).

Conclusion: Districts with a high burden of cataract blindness should be provided with free or highly subsidized cataract outreach programmes with effective publicity and high quality surgery.

Key words: cataract blindness, Igabi, Nigeria

\section{INTRODUCTION}

Cataract is a major cause of blindness worldwide. It is responsible for between 16 to 20 million blind people globally. ${ }^{1}$ As such, the elimination of cataract blindness is a major component of the World Health Organization (WHO) global coalition for the elimination of avoidable blindness by the year 2020: VISION 2020 - The Right to Sight. ${ }^{1}$
A prominent strategy for the Vision 2020 control of cataract blindness is the provision of cataract surgical services through outreach clinics, to remote areas. This way, the major obstacle of distance as a barrier to the uptake of cataract services will be overcome. The National Eye Centre (NEC), Kaduna, Nigeria, a government-owned tertiary eye hospital, plans to establish community-based cataract surgical services in the remote rural communities within its immediate vicinity in Igabi Local Government Area. The farthest community in this local government area (LGA) is within a 100-kilometre radius of the National Eye Centre, Kaduna; though travelling to the hospital may take several hours due to poor access roads, especially during the rainy season.

In order to effectively plan for such outreach services, a population-based survey, similar to the recently introduced WHO rapid assessment of cataract surgical services (RACCS), ${ }^{2}$ was conducted in the rural communities of the LGA. The survey was to determine the magnitude of cataract blindness, cataract surgical coverage, and the barriers to the uptake of cataract surgery in these communities. The survey was meant to provide baseline data on cataract blindness in the area, in order to determine the most appropriate cataract surgical services for these communities.

\section{MATERIALS AND METHODS}

The study was a cross-sectional survey of persons 40 years and older in Igabi LGA of Kaduna State, Nigeria. It was conducted in March 2001. A minimum sample size of 2400 was determined for the survey. This was based on an assumed prevalence of cataract blindness of $3.6 \%$ amongst persons 40 years and older (as reported in a similar survey of a rural community in northern Nigeria) ${ }^{3}$ and a maximum tolerable error of one per cent (1\%) with $95 \%$ confidence. A design effect of 1.5 was applied because of the cluster sampling technique used.

The study population was selected using a two-stage cluster random sampling method. In the first sampling stage, 30 clusters/villages were randomly selected from 120 villages in the local government area. The selection was effected using

${ }^{*}$ Correspondence: Dr. M Mansur Rabiu, Community Ophthalmology Department, National Eye Centre, PMB 2267, Kaduna, Nigeria;

Email:mrabiu@hotmail.com 
probability proportional to size (PPS). The bigger villages in the LGA with populations greater than 10,000 were excluded so as to ensure that only rural communities in the LGA were surveyed. In the $2^{\text {nd }}$ sampling stage, 80 persons aged $40 \mathrm{yrs}$ and over were randomly chosen from each of the selected clusters/villages. This was achieved by randomly selecting a direction from the centre of each village and selecting all households in that direction and sampling all eligible persons in household until the requisite number of 80 persons was attained.

A door-to-door registration and examination of all eligible subjects were conducted. The name, age, sex and occupation of all eligible persons were recorded. They were further asked if they had had cataract surgery or couching procedures. This was confirmed by examining the eye(s) for aphakia, or pseudophakia with a pen torch. Then the presenting visual acuity was tested for each eye; persons with aphakia had vision checked with +10 spectacles. All eyes with vision less than $6 / 18$ were retested with a pin hole. The ophthalmic nurses did the visual acuity testing with a modified ' $E$ ' Snellen chart (optotypes $6 / 60$ and $6 / 18$ only) placed at 6 metres in available outdoor light. Only one chance per optotype was given to subjects in reading of the charts.

All persons with vision less than 3/60 in any of the eyes were further examined by the ophthalmologist to find the likely principal cause of blindness in each eye using the operational definitions stated below. Blindness was defined as per the WHO definition of a presenting vision of less than $3 / 60$ in the better eye with best available correction. The ophthalmologist used a pen torch and direct ophthalmoscope to specifically examine for cataract, corneal opacity, uncorrected aphakia, retinal disease and glaucoma, using the following operational definitions. Couching is any traditional form of treating cataract that does not involve non-touch technique. Cataract refers to dense lens opacity that can explain a vision of less than 3/60. Corneal opacity refers to dense corneal opacity that can explain a visual loss of less than $3 / 60$. Glaucoma is defined as a cup/disc ratio of 0.9 or more, or if the media was not clear enough for optic disc assessment, a non-reactive pupil to light reflex in absence of definite causes like posterior synaechae. Uncorrected aphakia refers to an aphakic eye whose vision can improve to better than 3/60 with an aphakic glass (+10 glasses). Retinal disease means any form of retinal, optic nerve or macular disease that can cause vision less than 3/60. Others refers to all other conditions not listed above that can explain visual loss.

Persons with cataract in one or both eyes were asked why they had not had cataract surgery; and they were subsequently referred to the National Eye Centre, Kaduna for surgery. All information was recorded in a specially designed recording form. The recording forms were scrutinized at the end of each day's work, and where necessary, villages were revisited to complete missing information. Data was edited accordingly and entered using Epi-info software by double entry to prevent errors in data entry. The data was subsequently analysed using the C-sample programme of the Epi-info software.

Before starting the fieldwork, the consent of the communities to be visited was obtained through their respective village heads and the health department of the local government area. The survey team consisting of 2 ophthalmologists and 4 ophthalmic nurses trained for two days. The training involved standardization of visual acuity testing and examination procedures; and processes of administering and filling the questionnaire.

\section{RESULTS}

A total of 2044 persons, aged 40 years and above, were examined in the 30 clusters/villages. These constituted $85.2 \%$ of the minimum sample size determined for the survey.

\section{Age-Sex Distribution}

Of the 2044 persons examined, 1073 (52.5\%) were men and 971 $(47.5 \%)$ were women. The age-sex pyramid of the sampled population is presented in figure 1 .

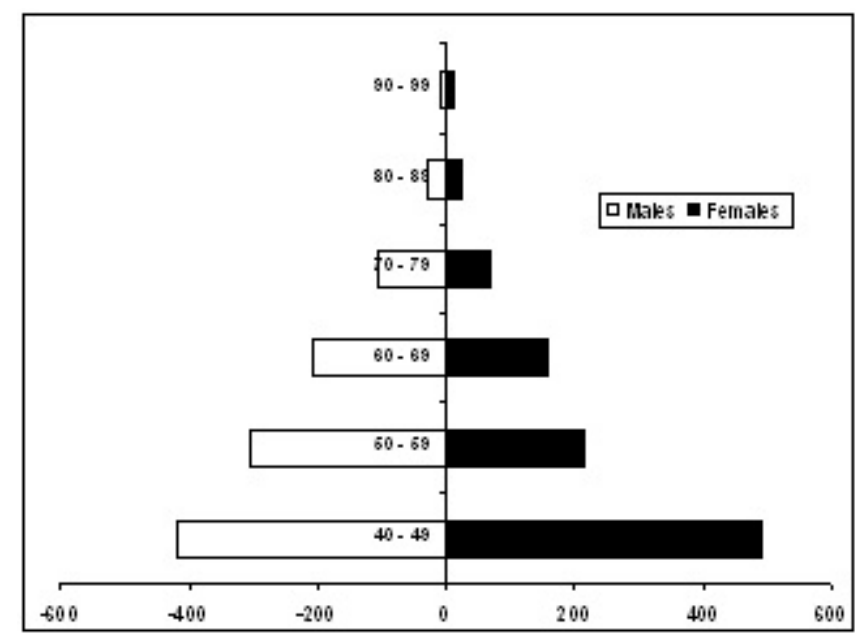

Figure 1. Age group by sex distribution of the sample population

\section{Prevalence of Blindness}

Eighty four (84) persons presented with a visual acuity (VA) of less than $3 / 60$ in the better eye, whereas 166 people presented with a VA of $3 / 60$ or better but less than $6 / 18$. The prevalence of bilateral blindness was $4.1 \% \quad(95 \%$ CI $3.2 \%-5.0 \%$, and that of low vision was $8.1 \%(95 \% \mathrm{CI}$ $6.9 \%-9.3 \%$ ) amongst the study population. The prevalence of blindness among the male patients was $4.66 \%$ (50/1073); while female patients had a prevalence of $3.50 \%$ (34/971). There was, however, no significant difference between the prevalence values of male and female patients. $\left(x^{2}=1.74, \mathrm{df}=1, \mathrm{P}\right.$ value $0.19)$. 
The prevalence of blindness, which increased with age across the different age groups, ranged from $0.88 \%$ to $16.36 \%$. Table 1 presents the distribution of blindness by age group and sex. The age and sex adjusted prevalence could not be calculated as the age and sex census data was not available.

The prevalence of unilateral blindness was $6.5 \%$ (CI 5.4\%$7.6 \%)$, There was a significant difference between the sexes, with males having a prevalence of $8 \%$ and females $4.8 \%$ (P value 0.00 ).

Table 1. Distribution of blindness by age group and sex

\begin{tabular}{lccc}
\hline $\begin{array}{l}\text { Age } \\
\text { groups }\end{array}$ & $\begin{array}{c}\text { Males } \\
\text { (prevalence \%) }\end{array}$ & $\begin{array}{c}\text { Females } \\
\text { (prevalence \%) }\end{array}$ & $\begin{array}{c}\text { Number of } \\
\text { Blind } \\
\text { (prevalence \%) }\end{array}$ \\
\hline $40-49$ & $7(1.7)$ & $1(0.20)$ & $8(0.88)$ \\
$50-59$ & $10(3.3)$ & $8(3.7)$ & $18(3.45)$ \\
$60-69$ & $15(7.3)$ & $18(11.3)$ & $33(9.02)$ \\
$70-79$ & $13(12.4)$ & $2(2.89)$ & $15(8.62)$ \\
$80-89$ & $4(13.3)$ & $5(20.0)$ & $9(16.36)$ \\
$90+$ & $1(14.3)$ & $-(0.00)$ & $1(5.26)$ \\
\hline Total & $50(4.66)$ & $34(3.50)$ & $84(4.11)$ \\
\hline
\end{tabular}

\section{Causes of Blindness}

Among the patients tested, cataract was the major cause of bilateral blindness, affecting $44(52.4 \%)$ out of 84 patients. Table 2 shows the other causes of bilateral blindness identified in the study.

Table 2. Distribution of causes of bilateral blindness

\begin{tabular}{lc}
\hline Causes & No. of people \\
\hline Cataract & $44(52.4)$ \\
Glaucoma & $10(11.9)$ \\
Couching & $10(11.9)$ \\
Corneal opacity & $8(9.5)$ \\
Retinal diseases & $3(3.6)$ \\
Uncorrected aphakia & $2(2.4)$ \\
Others & $7(8.3)$ \\
\hline Total & $84(100)$ \\
\hline
\end{tabular}

The prevalence of bilateral cataract blindness was found to be $2.2 \%$ (95CI $1.6 \%-2.8 \%)$ amongst the sampled population. This means that between 1,072 and 1,876 people in the LGA have cataract blindness in both eyes and are in urgent need of cataract surgery. The male patients had a prevalence of $2.2 \%$ and female patients had a prevalence of $2.1 \%$. However the difference between the prevalence in males and females was not statistically significant $\left(x^{2}=0.08, \mathrm{df}=1, \mathrm{P}\right.$ value $=0.78$ ).

The prevalence of unilateral cataract blindness was $3.5 \%$ (CI $2.7 \%-4.3 \%$ ). This means that between 1809 and 2881 people in the LGA had cataract blindness in one eye.
There were, therefore, at least 2,881 people in the LGA with cataract blindness in one or both eyes, who were in urgent need of cataract surgery.

\section{Cataract Surgical Coverage}

A total of 115 people were identified to have had cataract blindness in one or both eyes (44 people with bilateral cataract and 71 with unilateral cataract), while 19 people $(12.8 \%)$ had had cataract surgery in one eye and 15 people $(10.3 \%)$ had had couching procedure in one eye. Operable cataract was identified as responsible for vision less than $3 / 60$. Thus, over $75 \%$ of the people requiring cataract surgery have neither had surgery nor couching.

Cataract surgical coverage for male patients was $14.4 \%$ while female patients had cataract surgical coverage of $10.2 \%$; this difference was not statistically significant $\left(x^{2}=0.59, \mathrm{df}=\right.$ $1, \mathrm{P}$ value 0.44$)$.

\section{Visual Outcome of Cataract Surgery or Couching}

Out of the 19 eyes previously operated for cataract, six (31.6\%) had a vision worse than $3 / 60$ post-operatively, while of the 15 eyes couched, twelve $(80 \%)$ had a vision of worse than $3 / 60$ after the couching. Table 3 shows the visual status of eyes by the type of intervention (i.e., cataract surgery or couching).

\begin{tabular}{lllll}
\multicolumn{6}{l}{ Table 3. Visual status of eyes by } & \multicolumn{4}{l}{$\begin{array}{l}\text { type of intervention } \\
\text { Intervention }\end{array}$} & $\begin{array}{l}\text { Normal vision } \\
6 / 6-6 / 18\end{array}$ & $\begin{array}{l}\text { Low vision } \\
>6 / 18-3 / 60\end{array}$ & Blind & Total \\
\hline $\begin{array}{l}\text { Cataract } \\
\text { surgery }\end{array}$ & $9(47.3)$ & $4(21.1)$ & $6(31.6)$ & $19(100)$ \\
Couching & $2(13.3)$ & $1(6.7)$ & $12(80.0)$ & $15(100)$ \\
\hline Total & $11(32.3)$ & $5(14.7)$ & $18(53.0)$ & $34(100)$ \\
\hline
\end{tabular}

\section{Barriers to Cataract Surgery}

Cost was the major identified barrier to seeking cataract surgery. Table 4 presents the patients' constraints to uptake of cataract surgery amongst the sampled population.

\begin{tabular}{lc} 
Table 4. Barriers to uptake of cataract surgery \\
\hline Barriers & No. of subjects (\%) \\
\hline Cannot afford & $80(69.6)$ \\
Can see with other eye (unilateral & $13(11.3)$ \\
cataract) & $5(4.3)$ \\
Fear of surgery & $5(4.3)$ \\
Not aware of place of treatment & $3(2.6)$ \\
No benefit & $1(0.9)$ \\
Distance from hospital & $8(7.0)$ \\
No response & \\
\hline Total & $115(100)$ \\
\hline
\end{tabular}




\section{DISCUSSION}

A participation rate of $85.6 \%$ was obtained during the survey because in five clusters some individuals refused examination.

It can be estimated from the survey that there are over 2,800 people with cataract blindness in one or both eyes needing cataract surgery in these rural communities, i.e., that are within a $100 \mathrm{~km}$ radius of a large eye care facility (i.e., The National Eye Centre, Kaduna). This number includes at least 1,072 bilaterally blind, and 1,809 with unilateral cataract. It is imperative that a community-based outreach service be established in these communities.

The cataract blindness prevalence of $2.2 \%$, and a cataract surgical coverage (CSC) of only $12.8 \%$, among people aged 40 years and above in the study, differ from the values obtained in other rural areas of Nigeria., ${ }^{3,45}$ This prevalence rate is also similar to a specific study of a rural community in northern Nigeria, where a cataract blindness prevalence of $3.6 \%$ and a CSC of $4.1 \%$ were reported amongst persons 40 years and above. $^{3}$ Also in the rural district of Karnakata State of India, a survey revealed that the age and sex adjusted prevalence of cataract blindness amongst persons 50 years and above varies from $1.58 \%$ to $7.24 \%$ across the rural districts. ${ }^{6}$ However, in more developed countries like China, the scenario seems better. Zhao et al. reported that in Shunyi district of Beijing, the cataract blindness prevalence was $1.63 \%$, and remarkably, the CSC was $56.36 \%$ amongst adults aged 50 and above. ${ }^{7}$

The high burden of cataract blindness with associated low CSC in this study area, where villages are within a $100 \mathrm{~km}$ radius from the National Eye Centre, Kaduna, is a cause for concern, as the centre provides regular and quality cataract surgical services at a fairly subsidized rate. Moreover, there is no significant waiting list, so patients do not have to wait for long before having their surgery done. In Nkhoma, Malawi, a similar situation was reported, where a study of 31 villages within a 10-mile radius of an affordable cataract surgery facility, revealed a blindness prevalence of $3.12 \%$ amongst persons 40 years and above; $62 \%$ of the blindness was due to cataract. In fact, the CSC was only $14.8 \%$. This means that only 1 in 7 people with bilateral cataract had received surgery. ${ }^{8}$ Distance from a cataract surgery facility has been one of the main factors identified as a barrier to the uptake of cataract surgery.

These studies have shown, however, that even if communities are close to an eye-care facility, other significant barriers still exist, such as the cost of surgery. Indeed, $69.5 \%$ of persons with cataract in this study could not afford cataract surgery. Most of the people in these poor communities earn less than 1 US dollar per day, thus the direct and indirect costs of accessing cataract surgical services are beyond their reach. As such, the provision of free or highly subsidized surgical services is the only way to provide eye care in such communities.
It is significant that some people $(4.3 \%$ of persons needing cataract surgery) in this study expressed fear of surgery as a barrier to the uptake of cataract surgery. These may be people who have a relation or a friend who has had cataract surgery with poor outcome. The study revealed that one-third of the eyes that have had cataract surgery remained blind. In fact, 'fear of surgery' was identified as a major obstacle to eye-care utilization by an international workshop on barriers to using eye-care services that held in Tamil Nadu, India in 1997. ${ }^{9}$ This emphasizes the need for high quality cataract services as a prerequisite for the success of any such service and indeed any eye-care programme.

Furthermore, it is worthy of note that some cataract patients in the study are not aware of where to seek treatment for cataract, despite the fact that the National Eye Centre, Kaduna is almost next door to their communities (less than $100 \mathrm{~km}$ ). This signifies the need for appropriate 'social marketing' of cataract services in the form of appropriate publicity. The use of trained community health workers in rural areas as cataract surgical rate workers $(\mathrm{CSRW})^{10}$ is a new innovation that may solve the problem of non-awareness of places that provide cataract surgical services among people who need the services. The CSRW are meant to identify cataract cases, enlighten them on the surgery and send them to an appropriate eye facility for surgery.

The limitation of this study includes the fact that the survey is mainly targeted cataract and cataract services, and so cannot adequately identify and estimate the magnitude of other causes of blindness, such as glaucoma, and other posterior segment diseases.

\section{CONCLUSION}

This study not only provides the baseline data for the commencement of cataract surgical services in rural communities, but also advocates that high quality services should be provided free or at highly subsidized rates, and should be well 'publicized'.

\section{ACKNOWLEDGEMENTS}

We wish to acknowledge the support of the management of the National Eye Centre, Kaduna for providing the funds for the survey.

\section{REFERENCES}

1. World Health Organization: Global Initiative for the elimination of avoidable blindness. WHO/PBL/97.61. Rev.2. pg. 1-10.

2. World Health Organisation. Rapid Assessment of Cataract Surgical Services. A package for data entry and analysis from population based rapid assessment WHO/PBL/01.84. 
3. Rabiu MM. Cataract blindness and barriers to uptake of cataract surgeries in a rural community of northern Nigeria. British Journal of Ophthalmology 2001; 85: 776-780.

4. Oluleye TS. Cataract blindness and barriers to cataract surgical intervention in three rural communities of Oyo State, Nigeria. Niger J Med 2004; 13(2): 156-60.

5. Abdullah UI. Prevalence of blindness in a north western Nigeria; rural population: a rapid assessment of cataract surgical services. Comm Eye H J 2004; 17(52): 63-4.

6. Limburg H, Kuwar R, Indrayan A, Sundaram KR. Rapid assessment of prevalence of cataract blindness at district level. Int J Epidemiol 1997; 26(5): 1049-54.

7. Zhao J, Sui R, Jia, L. et al. Prevalence of cataract and surgical coverage among adults aged 50 or above in Shunyi district of Beijing, China. Chung Hua Yen Ko Tsa Chih 2001; 37(1): 3-8.

8. Eloff J, Foster A. Cataract surgical coverage: results of a population-based survey at Nkhoma, Malawi. Ophthalmic Epidemiology, 2000; 7(3): 219-21.

9. Fletcher A. Barriers to using eye services and recommendation to improve uptake research finding and international workshop recommendation. Madurai. India. 1998: 27

10. Geert Vanneste. Breaking down barriers. How to increase the cataract surgical rate: A practical guide for eye units in developing countries. Christoffel Blindenmission, January 2001: 25. 